\title{
HOW DOES SPORT AFFECT MENTAL HEALTH? AN INVESTIGATION INTO THE RELATIONSHIP OF LEISURE-TIME PHYSICAL ACTIVITY WITH DEPRESSION AND ANXIETY
}

( ) University School of Physical Education in Wroclaw

original paper

DOI: https://doi.org/10.5114/hm.2019.78539

\section{KATJA SIEFKEN ${ }^{1}$, ASTRID JUNGE ${ }^{2}$, LENA LAEMMLE ${ }^{2}$}

${ }^{1}$ University of South Australia, Adelaide, Australia

${ }^{2}$ MSH Medical School Hamburg, Hamburg, Germany

\section{ABSTRACT}

Purpose. Physical activity (PA) can protect from developing mental disorders. Knowledge regarding optimal PA intensity, type, context, and amount for mental health is sparse. This study aims to determine the relationship between leisure-time PA and prevalence of depression and anxiety, and whether associations vary by PA amount, context, intensity, and type.

Methods. Overall, 682 recreational athletes (age: $M=33.0$ years; $S D=12.4 ; 53 \%$ female) completed a questionnaire on personal characteristics related to their leisure-time PA behaviour, the Centre of Epidemiologic Studies Depression Scale (CES-D), and the Generalized Anxiety Disorder (GAD-7) scale. Effect sizes (Cohen's d, $\eta^{2}$ partial) and power analyses using G*Power were applied.

Results. Individuals who met PA recommendations $(\mathrm{n}=596)$ reported lower depression scores $(M=11.69 ; S D=8.57)$ than those who did not meet the recommendations $(n=86 ; M=12.88 ; S D=8.73)$. Lowest depression and anxiety scores were observed for indoor team athletes $(M=10.75 ; S D=7.66$ and $M=2.94 ; S D=3.03$, respectively), followed by outdoor individual athletes $(M=11.17 ; S D=9.41$ and $M=3.39 ; S D=3.50$, respectively). Under specific conditions, high depression scores were noted for vigorous-intensity PA levels $(M=20.5 ; S D=4.94)$.

Conclusions. Meeting the WHO PA recommendations (moderate-intensity PA $150 \mathrm{~min} /$ week) reduces symptoms of depression and anxiety. However, no linear dose-response relation was observed. No differences were found between in- and outdoor or team and individual sports but between sport disciplines. Prospective research to evaluate the reasons is needed. Key words: sport, recreational athletes, psychological disorders, mental wellbeing

\section{Introduction}

Mental disorders are the core health challenge of the $21^{\text {st }}$ century [1] and contribute substantially to the global disease burden [2]. Addressing mental health is a global public health concern, and identifying and characterizing protective factors for mental health is essential for overcoming leading health challenges.

Regular physical activity (PA) has been shown to be associated with several benefits to overall physical health [3]. Epidemiological research suggests that PA can also influence mood [4], positive mental health, symptoms of depression and anxiety [5, 6]. Whilst mental health benefits of PA have been referenced in recent guidelines, to date insufficient evidence pre- cludes conclusions about the minimal or optimal types or amounts of PA for mental health' [7, p. G8-39]. The broad domains of PA include occupational, transport, domestic, and leisure [8]. Understanding the impact of leisure-time PA on mental health is focus of the present study. Leisure-time physical activities are those that are not required as essential activities of daily living and are performed at the discretion of the person [7]. These include activities such as sports, exercising, and recreational walking.

PA and mental health

A recent meta-analysis with 37 trials suggests that exercise may have a moderate-sized favourable asso-

Correspondence address: Katja Siefken, Alliance for Research in Exercise, Nutrition and Activity (ARENA), School of Health Sciences, University of South Australia, Internal Post Code CEA-14, GPO Box 2471, Adelaide SA 5001, Australia, e-mail: katja.siefken@unisa.edu.au

Received: July 31, 2017

Accepted for publication: September 8, 2018

Citation: Siefken K, Junge A, Laemmle L. How does sport affect mental health? An investigation into the relationship of leisure-time physical activity with depression and anxiety. Hum Mov. 2019;20(1):62-74; doi: https://doi.org/10.5114/hm.2019.78539. 
ciation with depression [9]. Another meta-meta-analysis shows that PA has significant reductive effects on anxiety and on depression [6]. The minimal or optimal amount, context, intensity, and type of PA for reducing anxiety and depression, however, remain unclear [10]. Studies focusing on athletes showed differences in the prevalence of depression between professionals and amateur athletes [11] or between different levels of play [12]. Whilst a body of research findings regarding mental health in elite athletes is available $[13,14]$, few empirical observations exist for recreational athletes.

\section{PA amount and mental health}

Guidelines from the World Health Organization (WHO) based on the metabolic equivalent of task (MET) values were used to assign the amount of PA [15]. The WHO guidelines recommend 150 min of moderate-intensity PA (from 3 to $<6$ METs) per week as a minimum amount for health enhancement. Alternatively, the guidelines recommend equivalent expenditure with vigorous intensity ( $\geq 6$ METs) PA (75 min/ week), or any combination of moderate and vigorous intensity activity that results in energy expenditure. MET values are defined as the ratio of the work metabolic rate to a standard resting metabolic rate (RMR) of $1.0 \mathrm{kcal} \cdot \mathrm{kg}^{-1} \cdot \mathrm{h}^{-1}$. One MET is considered the RMR or the energy cost of a person at rest [16]. The optimal threshold PA volume for mental health benefits was reported to be $2.5-7.5 \mathrm{~h} /$ week [17]. This minimal suggested amount of PA is in line with the global PA recommendations issued by the WHO.

\section{PA context and mental health}

We distinguish PA context by indoor and outdoor activities. Findings from a systematic review suggest that outdoor activities are associated with more positive effects on self-reported mental wellbeing than indoor activities [18]. This systematic review indicates that exercising outdoors is associated with greater feelings of revitalization, and positive engagement, decreases in tension, confusion, anger, and depression, and increased energy [18]. Equally, Mitchell [19] found that each additional use of natural environment per week was associated with a $6 \%$ lower risk of poor mental health. Repeated exercise in nature was reported to be connected to better emotional wellbeing [20]. The hypothesis of added beneficial effects to be gained from exercising outdoors has generated considerable interest, but there is a paucity of high quality evidence on which to base recommendations. Outdoor activities are subject to seasonality and poor or extreme weather conditions which have been identified as a barrier to participation in PA among various populations [21]. Therefore, indoor PA opportunities during the cold and/or hot months may foster regular PA behaviours year round and thus enhance mental wellbeing.

\section{PA intensity and mental health}

PA intensity is often expressed in METs, with energy expenditure measured in units of resting energy expenditure [16]. PA levels equivalent to $150 \mathrm{~min} /$ week of moderate-intensity activity or $75 \mathrm{~min} /$ week of vigorous activity (approximately 600 MET-min/ week) are considered an important target for health benefits [22]. The protective physical health effects are expected to continue at higher levels [22], whilst the protective mental health effects are largely unknown. On a scale relative to an individual's personal capacity, moderate-intensity PA is usually a 5 or 6 on a scale of $0-10$, whilst vigorous-intensity is usually a 7 or 8 [15]. Regarding PA intensity, research has found incoherent results. Although studies including clinical populations showed that vigorous PA $(\geqslant 6$ METs) was most effective in reducing depression [23], research from general populations found that even low doses of PA were associated with lower depression levels [6, 24]. Mutrie and Kannah [25] reported that depression score decreased with each additional episode of vigorous leisure activity in both men and women, but among men the decrease in depression score with moderate leisure activity was reversed if a lot of moderate activity was undertaken. In another study, mainly vigorous-intensity PA was associated with life satisfaction, and reduced depression [26]. Nixdorf et al. [11], as well as Junge and Feddermann-Demont [12] found that depression rates in elite athletes were not higher than in the general population, and Asztalos et al. [10] conclude in their population-based study with 6803 adults that the PA-mental health relationship is always positive, independent of intensity levels. As such, findings on intensity of PA behaviour for optimal mental health are ambivalent.

\section{PA type and mental health}

Within the type of leisure-time PA, 4 different modes of PA can be differentiated: team sport, individual sport, organized but non-competitive PA, and non-organized PA [27]. In the present study, we examine the differences 
K. Siefken, A. Junge, L. Laemmle, Leisure-time physical activity and mental health

of the effects of team and individual sport on mental health. In a systematic review of the psychological benefits of participation in sport for recreation in adults, team sport seemed to be particularly associated with improved psychosocial health outcomes owing to the social nature of the participation [28]. Another study, with 148 male students in India, equally suggests that team athletes show better mental health outcomes than individual athletes [29]. On the basis of the conceptual model for the effects of PA on mental health outcomes, individuals that prefer to engage in individual sports may equally derive mental health benefits, depending on individual preferences and character traits. In professional athletes, higher average depression scores were found in individual rather than in team sport athletes [30, 31], while one study found no difference in levels of depression between individual and team athletes [32]. Social support and relationships in team sports may protect the athlete from developing depressive symptoms.

\section{Material and methods}

The total of 1045 individuals that identify themselves as recreational athletes filled in a questionnaire; 682 subjects completed the questionnaire (response rate: $65.2 \%)$. The study represents a cross-sectional approach. It took place between October 2014 and January 2015. Data were collected through an electronic data collection system (online survey) and a paper/pencil version of the questionnaire.

\section{Survey}

\section{Centre for Epidemiologic Studies Depression Scale (CES-D)}

Whilst various instruments that measure depression levels exist, the Centre for Epidemiologic Studies Depression Scale (CES-D) has been stated the most balanced and representative test among depression inventories, which also include scales by Beck, Hamilton, and Zung [33]. The CES-D is a depression scale that was developed by the National Institute of Mental Health in the USA specifically for use in epidemiological studies [34]. Participants self-assess depressive symptoms by stating the frequency of acute and enduring strain, affects, and negative cognitive patterns of thought in the previous 7 days. The sum over the 20 items indicates the level of depression. A standard cut-off value of 16 has been established to screen a high risk of depression [35], which is clinically simi- lar to mild depression (score: 16-26), whereas a score of 27 or more can be seen as moderate to severe depression. Hautzinger [36] translated the CES-D into the German language and named it Allgemeine Depressions Skala (ADS), which is similar to but not identical with the CES-D. As suggested by Nixdorf et al. [11], the score of 23 was set as the cut-off value for major depressive symptoms.

\section{Generalized Anxiety Disorder 7-item (GAD-7)}

The GAD-7 is a 7-item questionnaire on symptoms of generalized anxiety disorders during the previous 2 weeks. The sum score is calculated by adding the answers to the 7 items on a 4 -point Likert scale (0-3), with scores ranging from 0 to 21 . The cut-off score is 10 or more for moderate and 15 or more for severe anxiety disorder.

\section{Physical activity}

PA was measured through different domains. The survey included questions on sport discipline, PA amount, PA intensity, PA type, PA context, and psychosocial aspects of PA. All questions relating to PA refer to leisure-time PA.

Amount was measured via the following question: 'In the past 12 months, how often did you engage in leisure-time PA on average per week?' Days/week and min/day could be chosen via drop-down menus. PA amount was treated as a 4-level categorical variable with the categories 'inactive,' 'insufficiently active,' 'meeting current PA recommendations,' and 'highly active,' as suggested by recent comparative risk assessment studies [37]. Since this study explores PA behaviour of recreational athletes, the 'inactive' group was removed from the analysis, and the following 3 categories were left: (1) 'insufficiently active,' (2) 'meeting current PA recommendations,' and (3) 'highly active'. Group 2 and 3 were combined, so that 2 categories: (1) 'insufficiently active' and (2) 'meeting current PA guidelines' remained.

Context was measured via the question 'Do you perform leisure-time PA indoors or outdoors?' with the following possible answers to choose from: (a) only outdoors; (b) mainly outdoors; (c) mainly indoors; d) only indoors.

Intensity was measured via the Borg scale rating. A drop-down menu (0-10) provided 10 intensity levels to choose from. Low-intensity was rated $0-4$, moderate intensity was rated 5 or 6 , and vigorous intensity was rated $7-10$. 
Type was measured via the question 'Do you exercise alone or in a team?' with the following possible answers to choose from: (a) alone; (b) with an often changing group (e.g. gym, aerobic classes); (c) with a rather consistent group/team (e.g. social running teams); (d) in a team. The perceived feeling of being part of a team was measured via the question 'Do you feel as part of a team in your key leisure-time PA?' with the possible answers: 'Yes' or 'No'. Joint aftersport activities were measured with the question 'Do you engage in after-sport activities with your co-athletes?' The following possible answers were to choose from: (a) never / very rarely; (b) rarely; (c) sometimes; (d) often; (e) very often / always.

\section{Data collection}

The questionnaire was made available both online and as a paper/pencil version. The online survey was accessible via various channels (posting in Facebook groups, XING sporting groups, announcements in major sport club newsletters, posting on sport club websites). Selected major sport clubs and sport institutions were cooperation partners and announced the study on their websites and in newsletters. Social media subpages (e.g. sport groups on Facebook and XING) posted the link to the questionnaire and encouraged participation. The printed paper/pencil questionnaire was disseminated to major local sport clubs, coaches, and sport associations. Posters and flyers with a quick response (QR) code enabled quick access to the online survey. They also pointed towards the paper/ pencil version, which was available in entrance halls and receptions of sport clubs and gyms. Coaches encouraged athletes to complete the survey prior to training sessions. Male and female recreational athletes (18-82 years of age) of any sport discipline filled in the survey. Completed paper/pencil surveys were manually entered into the digital database. The inclusion criteria were to exercise on a regular basis and to be at least 18 years old. All data were processed with the use of Excel and SPSS 23 software.

\section{Sample description}

Table 1 provides the key characteristics of the athletes that took part in the study.

\section{Statistical analysis}

Descriptive statistics were calculated as mean $\pm S D$ with the IBM SPSS Statistics software V23.0 (IBM
Table 1. Sample description

\begin{tabular}{|c|c|c|}
\hline Characteristics & $n$ & $\%$ \\
\hline \multicolumn{3}{|l|}{ Participants } \\
\hline Total & 1045 & 100 \\
\hline Completed & 682 & 65.2 \\
\hline Age & $33(S D: 12.4)$ & \\
\hline \multicolumn{3}{|l|}{ Sex } \\
\hline Male & 327 & 48 \\
\hline Female & 354 & 52 \\
\hline \multicolumn{3}{|l|}{ Occupation } \\
\hline Work & 391 & 57.3 \\
\hline Studying & 254 & 37.2 \\
\hline Housework & 11 & 1.6 \\
\hline Child raising & 11 & 1.6 \\
\hline None & 15 & 2.2 \\
\hline \multicolumn{3}{|l|}{ PA amount } \\
\hline Physically active* & 596 & 87.4 \\
\hline Physically inactive** & 86 & 12.6 \\
\hline \multicolumn{3}{|l|}{ Context } \\
\hline Outdoor & 461 & 76.6 \\
\hline Indoor & 221 & 32.4 \\
\hline \multicolumn{3}{|l|}{ Intensity } \\
\hline Vigorous-intensity PA & 524 & 76.8 \\
\hline Moderate-intensity PA & 151 & 22.1 \\
\hline Low-intensity PA & 7 & 1.0 \\
\hline \multicolumn{3}{|l|}{ Type } \\
\hline Exclusively individual sport & 346 & 50.7 \\
\hline Exclusively team sport & 119 & 17.4 \\
\hline Both individual and team sport & 217 & 31.8 \\
\hline \multicolumn{3}{|l|}{ Sport disciplines } \\
\hline Mixed & 341 & 50.0 \\
\hline Soccer & 88 & 12.9 \\
\hline Running & 65 & 9.5 \\
\hline Triathlon & 60 & 8.8 \\
\hline Cycling & 25 & 3.7 \\
\hline Hockey & 24 & 3.5 \\
\hline Basketball & 19 & 2.8 \\
\hline Gym workout & 15 & 2.2 \\
\hline Yoga/Pilates & 12 & 1.8 \\
\hline Volleyball & 10 & 1.5 \\
\hline Dancing & 7 & 1.0 \\
\hline Tennis & 7 & 1.0 \\
\hline Aerobics & 4 & 0.6 \\
\hline Rowing & 2 & 0.3 \\
\hline Swimming & 2 & 0.3 \\
\hline Squash & 1 & 0.1 \\
\hline
\end{tabular}




\section{HUMAN MOVEMENT}

K. Siefken, A. Junge, L. Laemmle, Leisure-time physical activity and mental health

Corp, Armonk, New York, USA). As the sample sizes strongly differed between groups, a focus was placed on effects size (Cohen's $d$ and $\eta^{2}$ partial), and power analyses with the use of $G^{*}$ Power [38] was applied. To analyse differences for more than 2 groups, ANOVA for independent samples was conducted after checking the assumptions of normality (Kolmogorov-Smirnov test) and variance homogeneity (Levene test). The significance level was set at $p<0.05$.

\section{Ethical approval}

The research related to human use has been complied with all the relevant national regulations and institutional policies, has followed the tenets of the Declaration of Helsinki, and has been approved by the authors' institutional review board or an equivalent committee.

\section{Informed consent}

Informed consent has been obtained from all individuals included in this study.

\section{Results}

Tree view diagrams (Figures 1-3) were developed and present a systematic analysis approach for the study.
The prevalence of depression and anxiety was compared with data of the general population in Germany. Effects of PA amount, context, intensity, and type on depression and anxiety were analysed.

Differences in anxiety levels were observed in team athletes, with higher anxiety scores for outdoor team athletes than for indoor team athletes (Table 2). Equally, outdoor team athletes reported higher depression levels than indoor team athletes. For individual and mixed athletes (practising both individually or in a team), no or only marginal such effects were observed for depression and anxiety levels in both indoor and outdoor contexts.

Adding the information whether the athletes met the PA recommendations or not (Table 3), athletes who did not meet the PA recommendations reported higher depression scores independent of whether they practised indoors or outdoors or whether they practised individually or in a team (small to high effects).

Nevertheless, there is one exception: for single indoor athletes, the amount of being active was irrelevant for their depression and anxiety levels. But outdoor team athletes who met the PA recommendations reported higher depression scores than outdoor team athletes who did not meet the PA recommendations. Overall, $73 \%$ of the outdoor team athletes who met

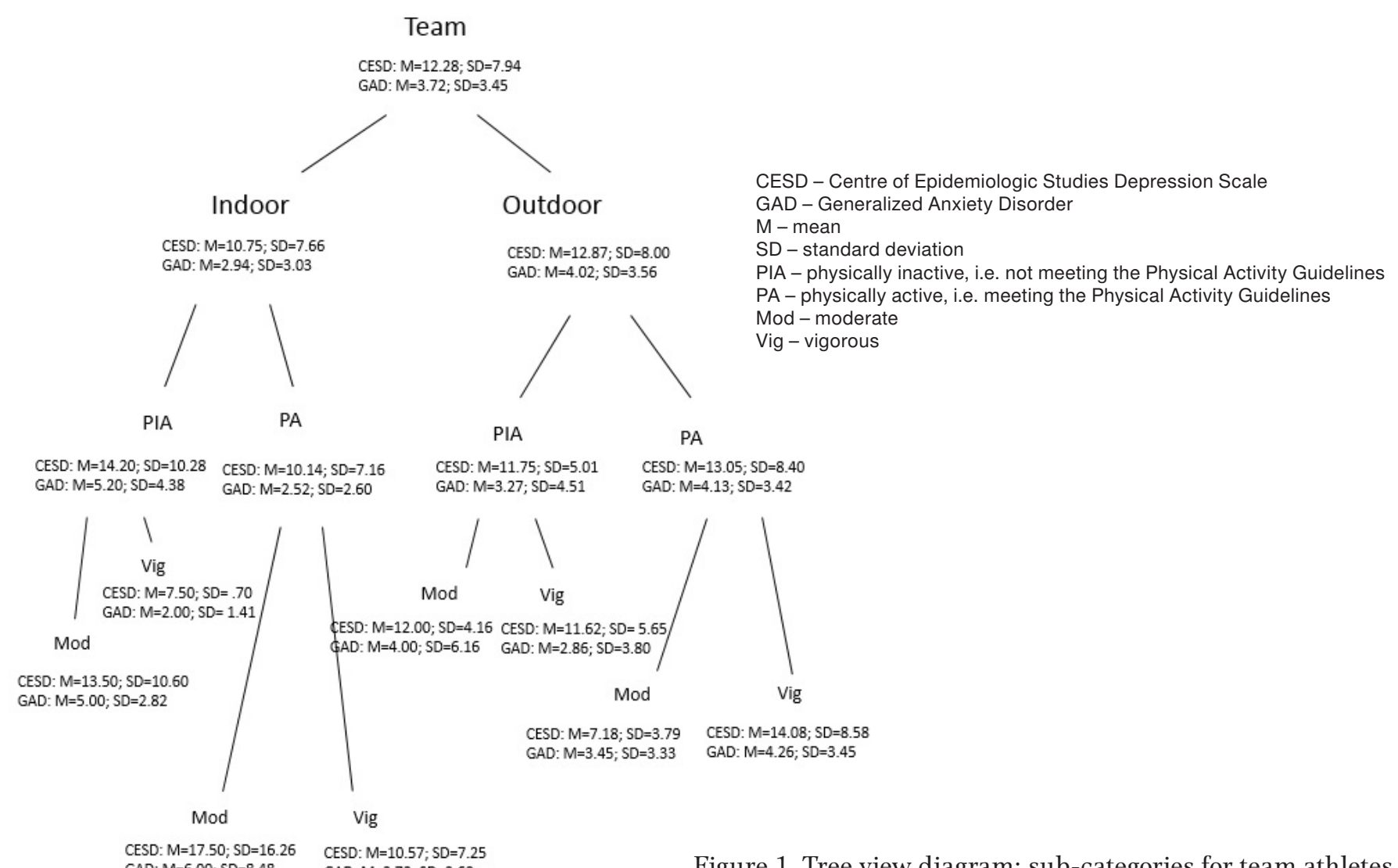




\section{Mixed}

CESD: $M=11.95 ; S D=8.23$ GAD: $M=3.42 ; S D=3.40$

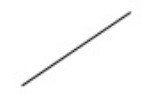

Indoor

CESD: $M=11.73 ; S D=6.9$

GAD: $M=3.41 ; S D=2.75$
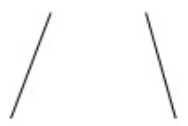

PIA

PA

$\begin{array}{ll}\text { CESD: } M=18.33 ; S D=5.13 & \text { CESD: } M=11.38 ; G A D: M=6.90 \\ \text { GAD: } M=3.00 ; S D=1.73 & \text { GAD: } M=3.43 ; S D=2.80\end{array}$

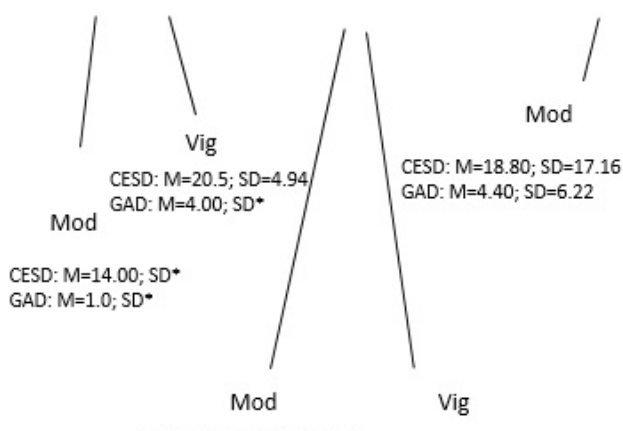

CESD: $M=13.87 ; S D=9.56 \quad$ CESD: $M=10.97 ; S D=6.41$ GAD: $M=3.50 ; S D=2.44 \quad$ GAD: $M=3.42 ; S D=2.87$
CESD - Centre of Epidemiologic Studies Depression Scale

GAD - Generalized Anxiety Disorder

$M-$ mean

SD - standard deviation

PIA - physically inactive, i.e. not meeting the Physical Activity Guidelines PA - physically active, i.e. meeting the Physical Activity Guidelines

Mod - moderate

Vig - vigorous

\section{Outdoor}

CESD: $M=12.07 ; S D=8.72$

GAD: $M=3.46 ; S D=3.63$

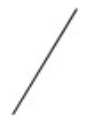

PIA
PA

CESD: $M=11.76 ; S D=8.39$

GAD: $M=3.36 ; S D=3.52$

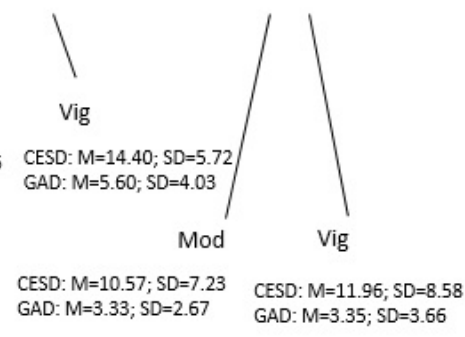

\section{Individual}

CESD: $M=11.62 ; S D=9.03$ $\mathrm{GAD}: \mathrm{M}=3.55 ; \mathrm{SD}=3.59$

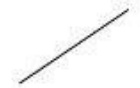

Indoor

CESD: $M=12.38 ; S D=8.32$ GAD: $M=3.82 ; S D=3.75$

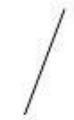

PIA

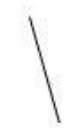

PA

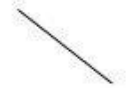

Outdoor
Figure 2. Tree view diagram: sub-categories for mixed athletes
CESD - Centre of Epidemiologic Studies Depression Scale

GAD - Generalized Anxiety Disorder

$M-$ mean

SD - standard deviation

PIA - physically inactive, i.e. not meeting the Physical Activity Guidelines

PA - physically active, i.e. meeting the Physical Activity Guidelines Mod - moderate

Vig - vigorous

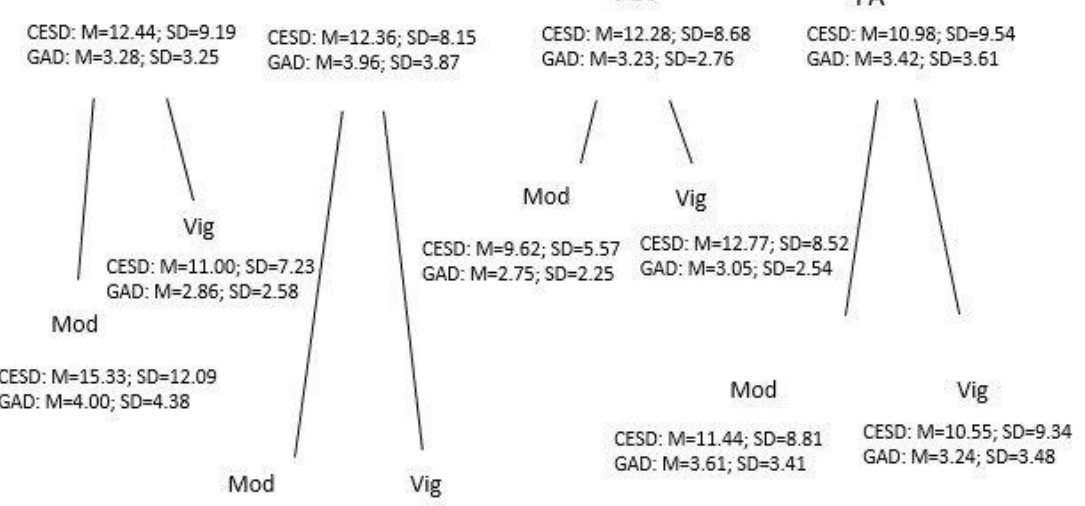

CESD: $M=13.76 ; S D=7.17$ GAD: $M=4.44 ; S D=3.63$
CESD: $M=11.18 ; S D=.41$

GAD: $M=3.39 ; S D=3.50$
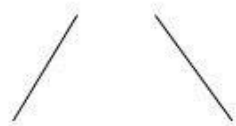

PIA

PA

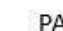

CESD: $M=10.98 ; S D=9.54$ 
K. Siefken, A. Junge, L. Laemmle, Leisure-time physical activity and mental health

Table 2. Descriptive statistics, test value, effect size, and power for type and context

\begin{tabular}{|c|c|c|c|c|c|c|c|c|c|c|c|c|c|}
\hline \multirow{2}{*}{ Type } & \multirow{2}{*}{ Context } & \multicolumn{6}{|c|}{ CES-D } & \multicolumn{6}{|c|}{ GAD } \\
\hline & & $n$ & $M \pm S D$ & $t$ & $d f$ & $d$ & $1-\beta$ & $n$ & $M \pm S D$ & $t$ & $d f$ & $d$ & $1-\beta$ \\
\hline \multirow{2}{*}{ Team } & Indoor & 33 & $10.75 \pm 7.66$ & \multirow{2}{*}{1.98} & \multirow{2}{*}{117} & \multirow{2}{*}{0.26} & \multirow{2}{*}{0.25} & 32 & $2.93 \pm 3.02$ & \multirow{2}{*}{1.98} & \multirow{2}{*}{114} & \multirow{2}{*}{0.66} & \multirow{2}{*}{0.88} \\
\hline & Outdoor & 86 & $12.87 \pm 8.00$ & & & & & 84 & $4.02 \pm 3.56$ & & & & \\
\hline \multirow{2}{*}{ Mixed } & Indoor & 60 & $11.73 \pm 6.96$ & \multirow{2}{*}{1.97} & \multirow{2}{*}{213} & \multirow{2}{*}{0.14} & \multirow{2}{*}{0.15} & 60 & $3.41 \pm 2.75$ & \multirow{2}{*}{1.97} & \multirow{2}{*}{212} & \multirow{2}{*}{$<0.01$} & \multirow{2}{*}{0.05} \\
\hline & Outdoor & 155 & $12.07 \pm 8.72$ & & & & & 154 & $3.46 \pm 3.63$ & & & & \\
\hline \multirow{2}{*}{ Single } & Indoor & 128 & $12.38 \pm 8.32$ & \multirow{2}{*}{1.96} & \multirow{2}{*}{344} & \multirow{2}{*}{0.11} & \multirow{2}{*}{0.18} & 123 & $3.82 \pm 3.75$ & \multirow{2}{*}{1.96} & \multirow{2}{*}{336} & \multirow{2}{*}{$<0.01$} & \multirow{2}{*}{0.05} \\
\hline & Outdoor & 218 & $11.17 \pm 9.41$ & & & & & 215 & $3.39 \pm 3.50$ & & & & \\
\hline
\end{tabular}

CES-D - Centre of Epidemiologic Studies Depression Scale, GAD - Generalized Anxiety Disorder

Table 3. Descriptive statistics, test value, effect size, and power for type, context, and amount

\begin{tabular}{|c|c|c|c|c|c|c|c|c|c|c|c|c|c|c|}
\hline \multirow{2}{*}{ Type } & \multirow{2}{*}{ Context } & \multirow{2}{*}{ Amount } & \multicolumn{6}{|c|}{ CES-D } & \multicolumn{6}{|c|}{ GAD } \\
\hline & & & $n$ & $M \pm S D$ & $t$ & $d f$ & $d$ & $1-\beta$ & $n$ & $M \pm S D$ & $t$ & $d f$ & $d$ & $1-\beta$ \\
\hline \multirow{2}{*}{ Team } & Indoor & $\begin{array}{r}\text { PA } \\
\text { PIA }\end{array}$ & $\begin{array}{r}28 \\
5\end{array}$ & $\begin{array}{l}10.14 \pm 7.16 \\
14.20 \pm 10.28\end{array}$ & 2.03 & 31 & 0.50 & 0.17 & $\begin{array}{r}27 \\
5\end{array}$ & $\begin{array}{l}2.51 \pm 2.60 \\
5.20 \pm 4.38\end{array}$ & 2.04 & 30 & 0.94 & 0.47 \\
\hline & Outdoor & $\begin{array}{l}\text { PA } \\
\text { PIA }\end{array}$ & $\begin{array}{l}74 \\
12\end{array}$ & $\begin{array}{l}13.05 \pm 8.40 \\
11.75 \pm 5.01\end{array}$ & 1.98 & 84 & 1.19 & 0.96 & $\begin{array}{l}73 \\
11\end{array}$ & $\begin{array}{l}4.13 \pm 3.42 \\
3.27 \pm 4.51\end{array}$ & 1.98 & 82 & 0.24 & 0.11 \\
\hline \multirow{2}{*}{ Mixed } & Indoor & $\begin{array}{l}\text { PA } \\
\text { PIA }\end{array}$ & $\begin{array}{r}57 \\
3\end{array}$ & $\begin{array}{l}11.38 \pm 6.90 \\
18.33 \pm 5.13\end{array}$ & 2.00 & 58 & 1.26 & 0.55 & $\begin{array}{r}57 \\
3\end{array}$ & $\begin{array}{l}3.43 \pm 2.80 \\
3.00 \pm 1.73\end{array}$ & 2.00 & 58 & 0.00 & 0.05 \\
\hline & Outdoor & $\begin{array}{l}\text { PA } \\
\text { PIA }\end{array}$ & $\begin{array}{r}145 \\
10\end{array}$ & $\begin{array}{l}11.76 \pm 8.39 \\
16.60 \pm 12.28\end{array}$ & 1.97 & 153 & 0.49 & 0.31 & $\begin{array}{r}144 \\
10\end{array}$ & $\begin{array}{l}3.36 \pm 3.52 \\
5.00 \pm 4.98\end{array}$ & 1.97 & 152 & 0.56 & 0.40 \\
\hline \multirow{2}{*}{ Single } & Indoor & $\begin{array}{r}\text { PA } \\
\text { PIA }\end{array}$ & $\begin{array}{r}103 \\
25\end{array}$ & $\begin{array}{l}12.36 \pm 8.15 \\
12.44 \pm 9.19\end{array}$ & 1.97 & 126 & 0.00 & 0.05 & $\begin{array}{l}98 \\
25\end{array}$ & $\begin{array}{l}3.96 \pm 3.87 \\
3.28 \pm 3.25\end{array}$ & 1.97 & 121 & 0.00 & 0.05 \\
\hline & Outdoor & $\begin{array}{r}\text { PA } \\
\text { PIA }\end{array}$ & $\begin{array}{r}186 \\
32\end{array}$ & $\begin{array}{l}10.98 \pm 9.54 \\
12.28 \pm 8.68\end{array}$ & 1.97 & 216 & 0.23 & 0.23 & $\begin{array}{r}185 \\
30\end{array}$ & $\begin{array}{l}3.42 \pm 3.61 \\
3.23 \pm 2.76\end{array}$ & 1.97 & 213 & 0.00 & 0.05 \\
\hline
\end{tabular}

CES-D - Centre of Epidemiologic Studies Depression Scale, GAD - Generalized Anxiety Disorder, PA - physically active (meeting PA recommendations), PIA - physically inactive (not meeting PA recommendations)

the PA recommendations were soccer players, $12.2 \%$ were field hockey players, and the remaining were mixed athletes.

Similar results emerged for anxiety; no difference in anxiety levels were detected for individual indoor athletes, mixed indoor athletes, or individual outdoor athletes.

Differentiating further whether the athletes practised to a moderate or vigorous degree (Table 4), higher depression scores were noted for vigorous-intensity PA practising athletes than for moderate-intensity PA practising athletes when practising indoors in a team and meeting the PA recommendations (strong effect).

Higher depression scores were also observed for outdoor vigorous-intensity practising team and individual athletes if they did not meet the PA recommendations (moderate to high effect).
However, under the following conditions, vigorousintensity practising athletes reported lower depression scores (small to moderate effect): if they practised on their own, indoors, and did not meet the PA recommendations; if they practised alone or within a team, indoors, and met the PA recommendations; if they practised outdoors, alone or within a team, and did not meet the PA recommendations. Even though the differences were small, again vigorous-intensity practising athletes reported lower depression scores for being active in a team, outdoors, not fulfilling the PA recommendations; or being active on their own, indoors, and fulfilling the PA guidelines.

Under 3 conditions a vigorous intensity level was associated with lower anxiety feelings; however, again in 3 cases a vigorous intensity level was associated with higher anxiety feelings (moderate to high effects). 
Table 4. Descriptive statistics, test value, effect size, and power for type, context, amount, and intensity

\begin{tabular}{|c|c|c|c|c|c|c|c|c|c|c|c|c|c|c|c|}
\hline \multirow{2}{*}{ Type } & \multirow{2}{*}{ Context } & \multirow{2}{*}{ Amount } & \multirow{2}{*}{ Intensity } & \multicolumn{6}{|c|}{ CES-D } & \multicolumn{6}{|c|}{ GAD } \\
\hline & & & & $n$ & $\mathrm{M} \pm \mathrm{SD}$ & $t$ & $d f$ & $d$ & $1-\beta$ & $n$ & $M \pm S D$ & $t$ & $d f$ & $d$ & $1-\beta$ \\
\hline \multirow{8}{*}{ Team } & \multirow{4}{*}{ Indoor } & \multirow{2}{*}{$\mathrm{PA}$} & Mod & 2 & $17.50 \pm 16.26$ & \multirow{2}{*}{2.05} & \multirow{2}{*}{26} & \multirow{2}{*}{0.56} & \multirow{2}{*}{0.11} & 2 & & & & & \\
\hline & & & Vig & 26 & $10.57 \pm 7.25$ & & & & & - & & & & & \\
\hline & & \multirow{2}{*}{ PIA } & Mod & 2 & & & & & & 2 & & & & & \\
\hline & & & Vig & 2 & & & & & & - & & & & & \\
\hline & \multirow{4}{*}{ Outdoor } & \multirow{2}{*}{ PA } & Mod & 11 & $7.18 \pm 3.78$ & \multirow{2}{*}{1.99} & \multirow{2}{*}{72} & \multirow{2}{*}{1.15} & \multirow{2}{*}{0.93} & 11 & $3.45 \pm 3.32$ & \multirow{2}{*}{1.99} & \multirow{2}{*}{71} & \multirow{2}{*}{0.33} & \multirow{2}{*}{0.17} \\
\hline & & & Vig & 63 & $14.07 \pm 8.58$ & & & & & 62 & $4.25 \pm 3.45$ & & & & \\
\hline & & \multirow{2}{*}{ PIA } & Mod & 4 & $12.00 \pm 4.16$ & \multirow{2}{*}{2.22} & \multirow{2}{*}{10} & \multirow{2}{*}{0.22} & 0.06 & 4 & $4.00 \pm 6.16$ & 2.26 & 9 & 0.42 & 0.09 \\
\hline & & & Vig & 8 & $11.62 \pm 5.65$ & & & & 0.00 & 7 & $2.85 \pm 3.80$ & $2 . \angle 0$ & 9 & 0.42 & 0.09 \\
\hline & & PA & Mod & 8 & $13.87 \pm 9.56$ & 200 & 55 & 039 & 0.17 & 11 & $3.45 \pm 3.32$ & 199 & 71 & 0.33 & 0.17 \\
\hline & & PA & Vig & 49 & $10.97 \pm 6.41$ & & & 0.39 & 0.17 & 62 & $4.25 \pm 3.45$ & 1.99 & 71 & 0.30 & 0.14 \\
\hline & & PIA & Mod & 1 & & & & & & 1 & & & & & \\
\hline & & PIA & Vig & 2 & & & & & & 2 & & & & & \\
\hline & & DA & Mod & 21 & $10.57 \pm 7.23$ & 107 & 142 & 012 & 0 & 8 & $3.50 \pm 2.44$ & 200 & 55 & 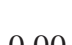 & 05 \\
\hline & & PA & Vig & 124 & $11.96 \pm 8.58$ & 1.97 & 143 & 0.13 & 0.08 & 49 & $3.42 \pm 2.87$ & 2.00 & 55 & 0.00 & 0.05 \\
\hline & & PIA & Mod & 5 & $18.80 \pm 17.16$ & 230 & 8 & 021 & 007 & 5 & $4.40 \pm 6.22$ & 230 & 8 & 010 & 005 \\
\hline & & PIA & Vig & 5 & $14.40 \pm 5.72$ & 2.30 & 8 & 0.31 & 0.07 & 5 & $5.60 \pm 4.03$ & 2.30 & 8 & 0.19 & 0.05 \\
\hline & & PA & Mod & 30 & $13.76 \pm 7.17$ & 198 & 101 & 026 & (2) & 27 & $4.44 \pm 3.63$ & 198 & 96 & 033 & 030 \\
\hline & $\mathrm{J}$ & $P A$ & Vig & 73 & $11.79 \pm 8.50$ & 1.98 & 101 & 0.26 & 0.22 & 71 & $3.78 \pm 3.97$ & 1.98 & 96 & 0.33 & 0.30 \\
\hline & & PIA & Mod & 9 & $15.33 \pm 12.09$ & 207 & 22 & 040 & 015 & 9 & $4.00 \pm 4.38$ & 207 & 22 & 063 & 0.30 \\
\hline & & PIA & Vig & 15 & $11.00 \pm 7.23$ & 2.07 & 22 & 0.40 & 0.13 & 15 & $2.86 \pm 2.58$ & 2.07 & 22 & 0.63 & 0.30 \\
\hline & & PA & Mod & 50 & $11.44 \pm 8.81$ & 197 & 182 & 011 & 010 & 49 & $3.61 \pm 3.41$ & 199 & 62 & 0 & 005 \\
\hline & & PA & Vig & 135 & $10.55 \pm 9.34$ & 1.91 & 183 & 0.11 & 0.10 & 135 & $3.24 \pm 3.48$ & 1.99 & 02 & 0.00 & 0.05 \\
\hline & & PIA & Mod & 8 & $8.62 \pm 5.57$ & 2 & 28 & 050 & 028 & 8 & $2.75 \pm 2.25$ & 205 & 26 & 050 & 21 \\
\hline & & PIA & Vig & 22 & $12.77 \pm 8.52$ & 2.04 & 28 & 0.59 & 0.28 & 20 & $3.05 \pm 2.54$ & 2.05 & 20 & 0.50 & 0.21 \\
\hline
\end{tabular}

CES-D - Centre of Epidemiologic Studies Depression Scale, GAD - Generalized Anxiety Disorder, PA - physically active (meeting PA recommendations), PIA - physically inactive (not meeting PA recommendations), Mod - moderate (3-6 MET), Vig - vigorous (> 6 MET)

Lower anxiety feelings were reported for those athletes who practised in a team, outdoors, and did not meet the PA recommendation; for individual indoor athletes who either met the PA recommendations or not.

Higher anxiety scores were reported by outdoor team athletes who met the PA recommendations; by mixed indoor athletes who met the PA recommendations; and by individual outdoor athletes who did not meet the PA recommendations. A small effect was observed for those who practised vigorously, alone, as well as in a team, outdoors, and not meeting the PA recommendations.

Comparing the effect of practising indoors or outdoors, in a team or individually, lowest depression scores were observed for indoor team athletes; they also reported lowest anxiety scores followed by outdoor single athletes (small effect; Table 5). Comparing the effect of meeting the PA recommendations in a team or individually, no differences emerged (Table 6) for anxiety or depression.

Finally, athletes did not differ in their depression scores with regard to joint after-sport activities $(F=0.34$; $p=0.85 ; \eta^{2}$ partial $\left.<0.01 ; 1-\beta=0.13\right)$ or perceived feeling of a team $\left(F<0.01 ; p=0.99 ; \eta^{2}\right.$ partial $<0.01$; $1-\beta=0.05)$, but differed to a small degree for sport discipline $\left(F=0.66 ; p=0.82 ; \eta^{2}\right.$ partial $=0.02 ; 1-\beta=$ $0.45)$; higher scores were observed for yoga/Pilates and cycling, as shown in Table 7.

A similar picture emerged for anxiety feelings: athletes did not differ in their anxiety feelings with regard to joint after-sport activities $(F=0.07 ; p=0.99$; $\eta^{2}$ partial $<0.01 ; 1-\beta=0.06$ ) or perceived feeling of a team $\left(F=0.04 ; p=0.85 ; \eta^{2}\right.$ partial $\left.<0.01 ; 1-\beta=0.05\right)$, but differed to a small degree for sport discipline 


\section{HUMAN MOVEMENT}

K. Siefken, A. Junge, L. Laemmle, Leisure-time physical activity and mental health

Table 5. Descriptive statistics, test value, effect size, and power for comparing effects of practising indoors/outdoors in a team or individually

\begin{tabular}{llrrrrrrrrrrrr}
\hline \multirow{2}{*}{ Context } & Type & \multicolumn{1}{c}{ CES-D } & \multicolumn{1}{c}{ GAD } \\
\cline { 3 - 13 } & & \multicolumn{1}{c}{$n$} & $M \pm S D$ & $t$ & $d f$ & $d$ & $1-\beta$ & $n$ & $M \pm S D$ & $t$ & $d f$ & $d$ & $1-\beta$ \\
\hline \multirow{2}{*}{ Outdoor } & Team & 86 & $12.87 \pm 8.00$ & \multirow{2}{*}{1.96} & 302 & 0.11 & 0.15 & 84 & $4.02 \pm 3.56$ & \multirow{2}{*}{1.96} & 297 & 0.33 & 0.73 \\
& Single & 218 & $11.17 \pm 9.41$ & & & & & 215 & $3.39 \pm 3.50$ & & & & \\
\hline \multirow{2}{*}{ Indoor } & Team & 33 & $10.75 \pm 7.66$ & \multirow{2}{*}{1.97} & \multirow{2}{*}{159} & 0.26 & 0.27 & 32 & $2.93 \pm 3.02$ & \multirow{2}{*}{1.97} & \multirow{2}{*}{153} & 0.33 & 0.38 \\
& Single & 128 & $12.38 \pm 8.32$ & & & & & 123 & $3.82 \pm 3.75$ & & & & \\
\hline
\end{tabular}

CES-D - Centre of Epidemiologic Studies Depression Scale, GAD - Generalized Anxiety Disorder

Table 6. Descriptive statistics, test value, effect size, and power for comparing effects of meeting the PA recommendations in a team or individually

\begin{tabular}{llcccccccccccc}
\hline \multirow{2}{*}{ Amount } & Type & \multicolumn{1}{c}{ CES-D } & \multicolumn{1}{c}{ GAD } \\
\cline { 3 - 13 } & & $n$ & $M \pm S D$ & $t$ & $d f$ & $d$ & $1-\beta$ & $n$ & $M \pm S D$ & $t$ & $d f$ & $d$ & $1-\beta$ \\
\hline \multirow{2}{*}{ PIA } & Team & 16 & $11.43 \pm 5.35$ & \multirow{2}{*}{1.99} & 71 & 0.14 & 0.08 & 15 & $3.33 \pm 3.99$ & \multirow{2}{*}{1.99} & $68<0.01$ & 0.05 \\
& Single & 57 & $12.35 \pm 8.83$ & & & & & 55 & $3.25 \pm 2.97$ & & & \\
\hline \multirow{2}{*}{ PA } & Team & 103 & $12.41 \pm 8.28$ & \multirow{2}{*}{1.96} & 390 & 0.11 & 0.17 & 101 & $3.78 \pm 3.37$ & \multirow{2}{*}{1.96} & $382<0.01$ & 0.05 \\
& Single & 289 & $11.48 \pm 9.08$ & & & & & 283 & $3.61 \pm 3.70$ & & & \\
\hline
\end{tabular}

CES-D - Centre of Epidemiologic Studies Depression Scale, GAD - Generalized Anxiety Disorder, PA - physically active (meeting PA recommendations), PIA - physically inactive (not meeting PA recommendations)

Table 7. Descriptive statistics for perceived feeling of a team, joint after-sport activities, and sport discipline

\begin{tabular}{|c|c|c|c|c|c|}
\hline \multirow{2}{*}{ Characteristics } & & \multicolumn{2}{|c|}{ CES-D } & \multicolumn{2}{|c|}{ GAD } \\
\hline & & $n$ & $M \pm S D$ & $n$ & $M \pm S D$ \\
\hline \multirow{2}{*}{ Perceived feeling of a team } & Yes & 451 & $11.75 \pm 8.49$ & 451 & $3.44 \pm 3.37$ \\
\hline & No & 231 & $12.03 \pm 8.81$ & 231 & $3.75 \pm 3.75$ \\
\hline \multirow{5}{*}{ Joint after-sport activities } & Never & 101 & $12.79 \pm 9.83$ & 101 & $4.09 \pm 4.14$ \\
\hline & Rarely & 127 & $12.08 \pm 10.17$ & 127 & $3.75 \pm 3.97$ \\
\hline & Sometimes & 288 & $11.43 \pm 7.33$ & 288 & $3.33 \pm 3.03$ \\
\hline & Often & 130 & $11.38 \pm 8.40$ & 130 & $2.71 \pm 2.85$ \\
\hline & Always & 36 & $13.28 \pm 8.89$ & 36 & $3.47 \pm 3.30$ \\
\hline \multirow{16}{*}{ Sport discipline } & Soccer & 88 & $13.10 \pm 7.52$ & 88 & $3.46 \pm 3.68$ \\
\hline & Basketball & 19 & $11.05 \pm 7.64$ & 19 & $3.21 \pm 2.84$ \\
\hline & Hockey & 24 & $13.21 \pm 8.98$ & 24 & $3.92 \pm 3.69$ \\
\hline & Volleyball & 10 & $10.80 \pm 5.01$ & 10 & $2.60 \pm 2.01$ \\
\hline & Tennis & 7 & $7.57 \pm 4.43$ & 7 & $2.80 \pm 2.16$ \\
\hline & Squash & 1 & 5.00 & 1 & 2.00 \\
\hline & Rowing & 2 & $14.50 \pm 0.495$ & 2 & $4.00 \pm 0.00$ \\
\hline & Swimming & 2 & $7.50 \pm 0.70$ & 2 & $4.00 \pm 5.65$ \\
\hline & Running & 65 & $9.77 \pm 7.08$ & 65 & $2.66 \pm 2.58$ \\
\hline & Cycling & 25 & $14.20 \pm 13.13$ & 25 & $4.75 \pm 4.90$ \\
\hline & Gym workout & 15 & $12.53 \pm 7.79$ & 15 & $3.27 \pm 3.05$ \\
\hline & Aerobic/spinning & 4 & $6.25 \pm 2.98$ & 4 & $1.50 \pm 0.57$ \\
\hline & Yoga/Pilates & 12 & $17.83 \pm 10.12$ & 12 & $6.55 \pm 4.78$ \\
\hline & Dancing & 7 & $9.57 \pm 5.38$ & 7 & $2.33 \pm 2.50$ \\
\hline & Triathlon & 60 & $11.38 \pm 10.56$ & 60 & $3.75 \pm 4.29$ \\
\hline & Mixed & 341 & $11.79 \pm 8.49$ & 341 & $3.59 \pm 3.46$ \\
\hline
\end{tabular}

CES-D - Centre of Epidemiologic Studies Depression Scale, GAD - Generalized Anxiety Disorder 
$\left(F=1.29 ; p=0.21 ; \eta^{2}\right.$ partial $\left.=0.03 ; 1-\beta=0.80\right) ;$ higher scores were observed for yoga/Pilates and cycling, as shown in Table 7 .

\section{Discussion}

\section{Key findings}

Despite the consensus that PA plays an important role in promoting mental health [39-42], little is known regarding the specific amount, context, intensity, or type of PA that may have an impact on mental health outcomes in recreational athletes. Through our study, we investigated the effects of these specific features. The findings are as follows:

1. Lowest depression and lowest anxiety scores were observed for indoor team athletes, followed by outdoor individual athletes (small effect; Table 2).

2. Those who did not meet the PA recommendations reported higher depression scores, independent of whether they practised inside or outside, or whether they practised individually or in a team (small to high effects; Table 3).

3. Higher depression scores were observed for vigorous-intensity PA practising athletes than for moderate-intensity PA practising athletes when practising indoors in a team and meeting the PA recommendations (strong effect, Table 4).

Key finding 1: Lowest depression and lowest anxiety scores were observed for indoor team athletes, followed by outdoor individual athletes (small effect; Table 2)

Whilst research suggests that outdoor activities are associated with less feelings of depression than indoor activities [19], and team athletes have lower prevalence rates of depression than individual athletes [43], our findings indicate that type and context are highly dependent on additional criteria and vary greatly. Team athletes showed higher anxiety and depression levels when practising outdoors than indoors, but individual athletes presented no difference in anxiety and depression levels when practising outdoors or indoors. Moderate to high differences in anxiety levels were observed in team athletes, with higher anxiety scores for outdoor team athletes than for indoor team athletes (Table 2).

We conclude that the optimal context of leisuretime PA (indoor/outdoor) is influenced by type (team/ individual) and other potential mechanisms manifesting at the neurobiological, psychosocial, and behav- ioural levels as suggested by Rebar et al. [6] and Lubans et al. [40]. The conceptual model for the effects of PA on mental health outcomes in children and adolescents, developed by Lubans et al. [40], illustrates these potential mechanisms of PA that impact mental health. This study endorses the proposed conceptual model as an approach to visualize under what conditions mental health changes that are due to PA behaviour may occur; we conclude that individual preferences, personality traits, and other psychosocial aspects are important and require attention when defining optimal PA features.

\section{Key finding 2: Those who did not meet the PA recommendations reported higher depression scores, independent of whether they practised inside or outside, or whether they practised individually or in a team (small to high effects; Table 3)}

The optimal amount of PA for mental health is unknown. Whilst several PA guidelines, PA recommendations, frameworks, and the necessary evidence for health benefits exist, these findings generally focus on physical health effects of PA. Little has been investigated in the field of the optimal amount of PA that specifically refers to mental health outcomes. Kim et al. [17] conclude that the optimal threshold volume for mental health benefits is 2.5-7.5 h/week of PA, which corresponds to the PA recommendations issued by WHO. Findings from this study confirm that athletes who meet the WHO PA recommendations report lower depression scores than those who do not meet the WHO PA recommendations. Importantly, the below key finding 3 illustrates that mental health outcomes are dependent on intensity and context levels, thus the generalization of $150 \mathrm{~min} /$ week of moderate-intensity activity or $75 \mathrm{~min} /$ week of vigorous activity must be reported carefully in context with promoting mental health and possibly be put in relation with intensity and context levels.

\section{Key finding 3: Higher depression scores were observed for vigorous-intensity PA practising athletes than for moderate-intensity $P A$ practising athletes when practising indoors in a team and meeting the PA recommendations (strong effect, Table 4)}

Just as context is influenced by type and other psychological features, defining the optimal PA intensity for positive mental health outcomes is ambiguous. 
K. Siefken, A. Junge, L. Laemmle, Leisure-time physical activity and mental health

Findings indicate that higher depression scores are more likely to be associated with indoor team athletes who practise in vigorous-intensity levels and meet the PA recommendations (Table 4). As seen in Table 4, the group for this specific feature is $n=26$ and after detailed data analysis it stands out that this group consists of $61.53 \%$ basketball players and $11.53 \%$ soccer players, and the remaining athletes are not definable for a certain sport discipline (mixed). This is an important finding, which highlights that basketball and indoor soccer players that practise 3-4 times per week (meeting PA recommendations) are at a special risk for developing depressive symptoms. The observation confirms that PA can also compromise mental health, especially when performed in a more intense manner [44]. Excessive exercise and overtraining syndrome are associated with mood deterioration (e.g. depression, anxiety, sleep disturbances, irritability) and it is therefore necessary to point out that the optimal intensity for PA depends not only on individual and psychosocial features, but also on the amount of PA. This finding also confirms research from general populations (e.g. Australia, USA), where low doses of PA are associated with lower depression levels [45]. The suggestion by Pedišić et al. [26], however, that mainly vigorous-intensity PA is associated with positive mental health cannot be confirmed. Rather, this study highlights the necessity for the detailed investigation of the separate specific features of leisure-time PA and points out that recommendations on optimal context, intensity, and type are highly dependent on individual preferences, personality traits, and other psychosocial factors.

\section{Limitations}

Because of the study's explorative cross-sectional design, several limitations were identified and it is not possible to make definitive causal inferences about the findings.

Only leisure-time PA was measured and habitual PA was disregarded in the survey. It remains unclear how and to what extent PA in other domains (occupational, domestic, transport) affects mental health outcomes (anxiety, depression).

A large majority of participants (50.0\%) were classified as 'mixed sport disciplines,' as they had indicated that they practised more than one sport discipline. When soccer players indicated that they also ran, they were categorized into 'mixed sport disciplines'. This led to a large proportion of 'mixed sport disciplines,' and a small share of the separate disciplines.
It was thus impossible to draw firm conclusions regarding the association of single sport disciplines and mental health outcomes. Besides, some of the findings from the sub-group analysis are unclear owing to the small sample size of sport disciplines.

The large majority of the sample were classified as 'physically active' ( $n=596 ; 87.4 \%$ ) (i.e. meeting the PA guidelines) and findings for the individuals not meeting the PA guidelines result from a small group of athletes only $(n=86 ; 12.6 \%)$.

The study suffers sampling bias, as only a few sport disciplines present sufficient numbers of participants for representative results. For the remaining sport disciplines, effects on anxiety and depression are largely unknown.

\section{Conclusions}

Meeting the WHO PA recommendations has been shown to reduce the risk of developing depressive and anxiety disorder symptoms. It is thus important to encourage the general population to be physically active. However, the dose-response relation is less clear. Our findings indicate that the approach 'the more, the better' is not necessarily appropriate with regard to mental health outcomes. This is an important finding for coaches, sport clubs, psychologists, and general practitioners who are encouraged to prescribe exercise to improve mental health. We conclude that the promotion of modest, achievable levels of PA that correspond to an athlete's interest, physical, and mental capacities, may lead to optimal mental health effects. Clearly, open and active communication between researchers, sport organizations, and associated stakeholders is essential.

To gain further knowledge for prevention programs, underlying mechanisms should be investigated further. Longitudinal study designs are recommended to draw causal and firm conclusions. Findings highlight the importance of analysing mode-specific PA levels in future studies, as drawing conclusions about the relationship between PA and mental health on the basis of total PA levels only may be misleading. Further, current PA guidelines do not take mode-specific PA characteristics into account and may thus not be most effective for achieving mental health benefits. The emerging evidence about differential effects of PA modes, however, calls for further research. We suggest to also investigate domain-specific PA in order to allow for a more detailed insight into PA behaviour. 


\section{Acknowledgements}

We thank Frank Weiland for his contribution in the data collection process.

\section{Disclosure statement}

No author has any financial interest or received any financial benefit from this research.

\section{Conflict of interest}

The authors state no conflict of interest.

\section{Funding source}

Funding for this study was provided by the Fédération Internationale de Football Association (FIFA).

\section{References}

1. Wittchen HU, Jacobi F, Rehm J, Gustavsson A, Svensson M, Jönsson B, et al. The size and burden of mental disorders and other disorders of the brain in Europe 2010. Eur Neuropsychopharmacol. 2011;21(9):655679; doi: 10.1016/j.euroneuro.2011.07.018.

2. Murray CJ, Vos T, Lozano R. Disability-adjusted life years (DALYs) for 291 diseases and injuries in 21 regions, 1990-2010: a systematic analysis for the Global Burden of Disease Study 2010. Lancet. 2013;380(9859): 2197-2223; doi: 10.1016/S0140-6736(12)61689-4.

3. Haskell WL, Blair SN, Hill JO. Physical activity: health outcomes and importance for public health priority. Prev Med. 2009;49(4):280-282; doi: 10.1016/j.ypmed.2009. 05.002 .

4. Ströhle A. Physical activity, exercise, depression and anxiety disorders. J Neural Transm. 2009;116(6):777784; doi: 10.1007/s00702-008-0092-x.

5. Rooney B, Smalley K, Larson J, Havens S. Is knowing enough? Increasing physical activity by wearing a pedometer. Wisconsin Med J. 2003;102(4):31-36.

6. Rebar AL, Stanton R, Geard D, Short C, Duncan MJ, Vandelanotte C. A meta-meta-analysis of the effect of physical activity on depression and anxiety in non-clinical adult populations. Health Psychol Rev. 2015;9(3): 366-378; doi: 10.1080/17437199.2015.1022901.

7. Physical Activity Guidelines Advisory Committee. Physical Activity Guidelines Advisory Committee Report, 2008. Washington, DC: U.S. Department of Health and Human Services; 2008.

8. Khan KM, Thompson AM, Blair SN, Sallis JF, Powell KE, Bull FC, et al. Sport and exercise as contributors to the health of nations. Lancet. 2012;380(9836):59-64; doi: 10.1016/S0140-6736(12)60865-4.

9. Cooney GM, Dwan K, Greig CA, Lawlor DA, Rimer J, Waugh FR, et al. Exercise for depression (review). Cochrane Database Syst Rev. 2013;9:CD004366; doi: 10.1002/14651858.CD004366.pub6.

10. Asztalos M, De Bourdeaudhuij I, Cardon G. The relationship between physical activity and mental health varies across activity intensity levels and dimensions of mental health among women and men. Public Health Nutr. 2010;13(8):1207-1214; doi: 10.1017/S1368980 009992825.

11. Nixdorf I, Frank R, Hautzinger M, Beckmann J. Prevalence of depressive symptoms and correlating variables among German elite athletes. J Clin Sport Psychol. 2013;7(4):313-326; doi: 10.1123/jcsp.7.4.313.

12. Junge A, Feddermann-Demont N. Prevalence of depression and anxiety in top-level male and female football players. BMJ Open Sport Exerc Med. 2016;2(1): e000087; doi: 10.1136/bmjsem-2015-000087.

13. Wolanin A, Gross M, Hong E. Depression in athletes: prevalence and risk factors. Curr Sports Med Rep. 2015; 14(1):56-60; doi: 10.1249/JSR.0000000000000123.

14. Rice SM, Purcell R, De Silva S, Mawren D, McGorry PD, Parker AG. The mental health of elite athletes: a narrative systematic review. Sports Med.2016;46(9):13331353; doi: 10.1007/s40279-016-0492-2.

15. World Health Organization. Global recommendations on physical activity for health. Geneva: WHO Press; 2010.

16. Ainsworth BE, Haskell WL, Herrmann SD, Meckes N, Bassett DR Jr, Tudor-Locke C, et al. 2011 Compendium of Physical Activities: a second update of codes and MET values. Med Sci Sports Exerc. 2011;43(8):15751581; doi: 10.1249/MSS.0b013e31821ece12.

17. Kim YS, Park YS, Allegrante JP, Marks R, Ok H, Ok Cho K, et al. Relationship between physical activity and general mental health. Prev Med. 2012;55(5):458-463; doi: 10.1016/j.ypmed.2012.08.021.

18. Thompson Coon J, Boddy K, Stein K, Whear R, Barton J, Depledge MH. Does participating in physical activity in outdoor natural environments have a greater effect on physical and mental wellbeing than physical activity indoors? A systematic review. Environ Sci Technol. 2011;45(5):1761-1772; doi: 10.1021/es102947t.

19. Mitchell R. Is physical activity in natural environments better for mental health than physical activity in other environments? Soc Sci Med. 2013;91:130-134; doi: 10.1016/j.socscimed.2012.04.012.

20. Pasanen TP, Tyrväinen L, Korpela KM. The relationship between perceived health and physical activity indoors, outdoors in built environments, and outdoors in nature. Appl Psychol Health Well Being. 2014;6(3): 324-346; doi: 10.1111/aphw.12031.

21. Tucker P, Gilliland J. The effect of season and weather on physical activity: a systematic review. Public Health. 2007;121(12):909-922; doi: 10.1016/j.puhe.2007.04.009.

22. World Health Organization. Global health risks. Mortality and burden of disease attributable to selected major risks. Geneva: WHO Press; 2009.

23. Craft LL, Landers DM. The effect of exercise on clinical depression and depression resulting from mental illness: a meta-analysis. J Sport Exerc Psychol. 1998;20(4): 339-357; doi: 10.1123/jsep.20.4.339.

24. Teychenne M, Ball K, Salmon J. Physical activity and likelihood of depression in adults: a review. Prev Med. 2008;46(5):397-411; doi: 10.1016/j.ypmed.2008.01.009. 
K. Siefken, A. Junge, L. Laemmle, Leisure-time physical activity and mental health

25. Mutrie N, Kannah MK. The importance of both setting and intensity of physical activity in relation to non-clinical anxiety and depression. Int J Health Promot Educ. 2007;45(1):24-32;doi:10.1080/14635240.2007.10708094.

26. Pedišić Ž, Greblo Z, Phongsavan P, Milton K, Bauman AE. Are total, intensity- and domain-specific physical activity levels associated with life satisfaction among university students? PLoS One. 2015;10(2):e0118137; doi: 10.1371/journal.pone.0118137.

27. Eime RM, Harvey JT, Sawyer NA, Craike MJ, Symons CM, Polman RC, et al. Understanding the contexts of adolescent female participation in sport and physical activity. Res Q Exerc Sport. 2013;84(2):157-166; doi: 10.1080/02701367.2013.784846.

28. Eime RM, Harvey JT, Brown WJ, Payne WR. Does sports club participation contribute to health-related quality oflife? Med Sci Sports Exerc. 2010;42(5):10221028; doi: 10.1249/MSS.0b013e3181c3adaa.

29. Patel MP, Chaudhari AD. Sports for positive mental health: a comparative study of mental health among individual athletes, team athletes and non-athletes. Int J Indian Psychol. 2014;1(4):34-43.

30. Nixdorf I, Frank R, Beckmann J. Comparison of athletes' proneness to depressive symptoms in individual and team sports: research on psychological mediators in junior elite athletes. Fron Psychol. 2016;7:893; doi: 10.3389/fpsyg.2016.00893.

31. Wolanin A, Hong E, Marks D, Panchoo K, Gross M. Prevalence of clinically elevated depressive symptoms in college athletes and differences by gender and sport. Br J Sports Med. 2016;50(3):167-171; doi: 10.1136/ bjsports-2015-095756.

32. Barmi MR. A comparative study of depression level among male athletic students engaged in individual and team sports versus male non-athletic students of schools from the second board of education in Karaj, Iran. Procedia Soc Behav Sci. 2011;30:352-356; doi: 10.1016/j.sbspro.2011.10.069.

33. Shafer AB. Meta-analysis of the factor structures of four depression questionnaires: Beck, CES-D, Hamilton, and Zung. J Clin Psychol. 2006;62(1):123-146; doi: 10.1002/jclp.20213.

34. Radloff LS. The CES-D scale: a self report depression scale for research in the general population. Applied Psychological Measurement. 1977;1(3):385-401; doi: 10.1177/014662167700100306.

35. Riediger M, Linden M, Wilms H-U. The German version of the CES-D scale as an instrument in gerontological research [in German]. Z Klin Psychol, Psychiatr Psychother. 1998;46(4):344-364.

36. Hautzinger M. The CES-D scale. A depression instrument for studies in the general population [in German]. Diagnostica. 1988;34(2):167-173.

37. Danaei G, Ding EL, Mozaffarian D, Taylor B, Rehm CJ, Murray J, et al. The preventable causes of death in the United States: comparative risk assessment of dietary, lifestyle, and metabolic risk factors. PLoS Med. 2009; 6(4):e1000058; doi: 10.1371/journal.pmed.1000058.

38. Faul F, Erdfelder E, Lang AG, Buchner A. G*Power 3: a flexible statistical power analysis program for the social, behavioral, and biomedical sciences. Behav Res Methods. 2007;39(2):175-191; doi: 10.3758/BF03193146.

39. Richards J, Jiang X, Kelly P, Chau J, Bauman A, Ding D. Don't worry, be happy: cross-sectional associations between physical activity and happiness in 15 European countries. BMC Public Health. 2015;15(1):53; doi: 10.1186/s12889-015-1391-4.

40. Lubans D, Richards J, Hillman C, Faulkner G, Beauchamp M, Nilsson M, et al. Physical activity for cognitive and mental health in youth: a systematic review of mechanisms. Pediatrics. 2016;138(3):e20161642; doi: 10.1542/peds.2016-1642.

41. Ten Have M, de Graaf R, Monshouwer K. Physical exercise in adults and mental health status findings from the Netherlands mental health survey and incidence study (NEMESIS). J Psychosom Res. 2011;71(5):342348; doi: 10.1016/j.jpsychores.2011.04.001.

42. Hamer M, Biddle SJH, Stamatakis E. Weekend warrior physical activity pattern and common mental disorder: a population wide study of 108,011 British adults. Int J Behav Nutr Phys Act. 2017;14(1):96; doi: 10.1186/ s12966-017-0549-0.

43. Frank R, Nixdorf I, Beckmann I. Depression among elite athletes: prevalence and psychological factors. Deutsche Zeitschrift für Sportmedizin. 2015;64:320326.

44. Peluso MAM, Guerra de Andrade LHS. Physical activity and mental health: the association between exercise and mood. Clinics. 2005;60(1):61-70; doi: 10.1590/ S1807-59322005000100012.

45. Loprinzi PD. Objectively measured light and moderateto-vigorous physical activity is associated with lower depression levels among older US adults. Aging Ment Health. 2013;17(7):801-805; doi: 10.1080/13607863. 2013.801066. 